\title{
Support-Intuitionistic Fuzzy Set: A New Concept for Soft Computing
}

\author{
Xuan Thao Nguyen \\ Department of applied Math- Informatics, Faculty of Information Technology, Vietnam national university of \\ agriculture (VNUA) \\ Email: thaonx281082@yahoo.com \\ Van Dinh Nguyen \\ Department of applied Math- Informatics, Faculty of Information Technology, Vietnam national university of \\ agriculture (VNUA) \\ Email: nvdinh2000@gmail.com
}

\begin{abstract}
Today, soft computing is a field that is used a lot in solving real-world problems, such as problems in economics, finance, banking... With the aim to serve for solving the real problem, many new theories and/or tools which were proposed, improved to help soft computing used more efficiently. We can mention some theories as fuzzy sets theory (L. Zadeh, 1965), intuitionistic fuzzy set (K Atanasov, 1986). In this paper, we introduce a new notion of support-intuitionistic fuzzy (SIF) set, which is the combination a intuitionistic fuzzy set with a fuzzy set. So, SIF set is a directly extension of fuzzy set and intuitionistic fuzzy sets (Atanassov). Then, we define some operators on support-intuitionistic fuzzy sets, and investigate some properties of these operators.
\end{abstract}

Index Terms- Support-Intuitionistic Fuzzy Sets, SupportIntuitionistic Fuzzy Relations, Support-Intuitionistic Similarity Relations

\section{INTRODUCTION}

Fuzzy set theory was introduced by L.Zadeh since 1965 [1]. Immediately, it became a useful method to study in the problems of imprecision and uncertainty. Since, a lot of new theories treating imprecision and uncertainty have been introduced. For instance, Intuitionistic fuzzy sets were introduced in1986, by K. Atanassov [2], which is a generalization of the notion of a fuzzy set. When fuzzy set give the degree of membership of an element in a given set, intuitionistic fuzzy set give a degree of membership and a degree of non-membership of an element in a given set. Then, the concept of fuzzy relations and intuitionistic fuzzy relations introduced [4],[5]. They together with their logic operators [10][11] and [12] are applied in many different fields.

Practically, lets' consider the following case: a customer (maybe a girl!) is interested in two products A and B. The customer has one rating of good (i) or bad (ii) for each of product. These ratings (i) and (ii) (known as intuitionistic ratings) will affect the customer's decision of which product to buy. However, the customer's financial capacity will also affect her decision. This factor is called support factor, with the value is between 0 and 1 . Thus, the decision of which product to buy are determined by the positive intuitionistic factors (i), negative intuitionistic factors (ii) and support factor (iii). If a product is considered good and affordable, it is the best situation for a buy decision. The most unfavorable situation is when a product is considered bad and not affordable (support factor is bad), in this case, it would be easy to refuse buying the product.

In this paper, we combine a intuitionistic fuzzy set with a fuzzy set. This raise a new concept called supportintuitionistic fuzzy (SIF) set. In which, there are three membership function of an element in a given set. The remaining of this paper was structured as follows: In section 2, we introduce the concept of supportintuitionistic; some properties of SIF sets will be appear in section 3. In section 4, we give some distances between two SIF sets. Finally, we construct the notions of support-intuitionistic fuzzy relation and investigate initially properties as reflexive, symmetric, transitive of its.

\section{SUPPORT-INTUITIONISTIC FUZZY SET}

Throughout this paper, $U$ will be a nonempty set called the universe of discourse. First, we recall some the concept about fuzzy set and intuitionistic fuzzy set.

Definition 1. [1] A fuzzy set $A$ on the universe $U$ is an object of the form

$$
A=\left\{\left(x, \mu_{A}(x)\right) \mid x \in U\right\}
$$

where $\mu_{A}(x)(\in[0,1])$ is called the degree of membership of $x$ in $A$.

Definition 2. [2]. An intuitionistic fuzzy (IF) set $A$ on the universe $U$ is an object of the form

$$
A=\left\{\left(x, \mu_{A}(x), \gamma_{A}(x)\right) \mid x \in U\right\}
$$

where $\mu_{A}(x)(\in[0,1])$ is called the "degree of membership of $x$ in $A$ ", $\gamma_{A}(x)(\in[0,1])$ is called the "degree of non-membership of $x$ in $A$ " and where $\mu_{A}$ and $\gamma_{A}$ satisfy the following condition: $\mu_{A}(x)+\gamma_{A}(x) \leq$ $1,(\forall x \in U)$. 
Now, we combine a intuitionistic fuzzy set with a fuzzy set. This raise a new concept called supportintuitionistic fuzzy (SIF) set. In which, there are three membership function of an element in a given set. This new concept is statement as following:

Definition 3. A support-intuitionistic fuzzy (SIF) set A on the universe $U$ is an object of the form

$$
A=\left\{\left(x, \mu_{A}(x), \gamma_{A}(x), \theta_{A}(x)\right) \mid x \in U\right\}
$$

where $\mu_{A}(x)(\in[0,1])$ is called the "degree of membership of $x$ in $A$ ", $\gamma_{A}(x)(\in[0,1])$ is called the "degree of non-membership of $x$ in $A$ ", and $\theta_{A}(x)$ is called the "degree of support-membership of $x$ in $A$ ", and where $\mu_{A}, \gamma_{A}$ and $\theta_{A}$ satisfy the following condition: $\mu_{A}(x)+\gamma_{A}(x) \leq 1,0 \leq \theta_{A}(x) \leq 1,(\forall x \in U)$.

The family of all support-intuitionistic fuzzy set in $U$ is denoted by $\operatorname{SIFS}(U)$.

\section{Some Remarks:}

+ The element $x_{*}$ is called "worst element" in $A$ iff $\mu_{A}\left(x_{*}\right)=0, \gamma_{A}\left(x_{*}\right)=1, \theta_{A}\left(x_{*}\right)=0$. The element $x^{*}$ is called "best element" in $A$ iff $\mu_{A}\left(x^{*}\right)=1, \gamma_{A}\left(x^{*}\right)=$ $0, \theta_{A}\left(x^{*}\right)=1$.

+ A support-intuitionistic fuzzy set reduce an intuitionistic fuzzy set when $\theta_{A}(x)=c \in[0,1] \forall x \in U$.

+ A support-intuitionistic fuzzy set is a fuzzy set when $\gamma_{A}(x)=1-\mu_{A}(x), \theta_{A}(x)=c \in[0,1] \forall x \in U$.

Here we define some special SIF sets:

+ A constant SIF set $(\widehat{\alpha, \beta, \theta})=\{(x, \alpha, \beta, \theta) \mid x \in U$, where $0 \leq \alpha+\beta \leq 1,0 \leq \theta \leq 1$.

+ the SIF universe set is $U=1_{U}=(\widehat{1,0,1})=$ $\{(x, 1,0,1) \mid x \in U\}$

+ the SIF empty set is $U=0_{U}=(\widehat{0,1,0})=$ $\{(x, 0,1,0) \mid x \in U\}$

+ For any $y \in U$, SIF sets $1_{y}$ and $1_{U-\{y\}}$ are, respectively, defined by: for all $x \in U$,

$$
\begin{aligned}
& \mu_{1_{y}}(x)=\left\{\begin{array}{l}
1 \text { if } x=y \\
0 \text { if } x \neq y
\end{array} \quad \gamma_{1 y}(x)=\left\{\begin{array}{l}
0 \text { if } x=y \\
1 \text { if } x \neq y
\end{array}\right.\right. \\
& \theta_{1_{y}}(x)=\left\{\begin{array}{l}
1 \text { if } x=y \\
0 \text { if } x \neq y
\end{array}\right. \\
& \mu_{1_{U-\{y\}}}(x)=\left\{\begin{array}{l}
0 \text { if } x=y \\
1 \text { if } x \neq y
\end{array} \gamma_{1_{U-\{y\}}}(x)=\left\{\begin{array}{l}
1 \text { if } x=y \\
0 \text { if } x \neq y
\end{array}\right.\right. \\
& \theta_{1_{U-\{y\}}}(x)= \begin{cases}0 & \text { if } x=y \\
1 & \text { if } x \neq y\end{cases}
\end{aligned}
$$

\section{SOME OPERATORS ON SIFS}

In this section, we introduce operators on $\operatorname{SIFS}(X U)$ as follows:

Definition 3. For all $A, B \in \operatorname{SIFS}(U)$,

- $A \subseteq B$ iff $\mu_{A}(x) \leq \mu_{B}(x), \quad \gamma_{A}(x) \geq \gamma_{B}(x)$ and $\theta_{A}(x) \leq \theta_{B}(x) \forall x \in U$.

- $A=B$ iff $A \subseteq B$ and $B \subseteq A$.

- $A \cup B=$

$$
\left\{\left(x, \mu_{A \cup B}(x), \gamma_{A \cup B}(x), \theta_{A \cup B}(x)\right) \mid x \in U\right\}
$$

where $\mu_{A \cup B}(x)=\max \left\{\mu_{A}(x), \mu_{B}(x)\right\}, \quad \gamma_{A \cup B}(x)=$ $\min \left\{\gamma_{A}(x), \gamma_{B}(x)\right\}, \theta_{A \cup B}(x)=\max \left\{\theta_{A}(x), \theta_{B}(x)\right\}$ $\forall x \in U$.

- $A \cap B=\left\{\left(x, \mu_{A \cap B}(x), \gamma_{A \cap B}(x), \theta_{A \cap B}(x), x \in U\right\}\right.$ where $\mu_{A \cap B}(x)=\min \left\{\mu_{A}(x), \mu_{B}(x)\right\}, \quad \gamma_{A \cap B}(x)=$ $\max \left\{\gamma_{A}(x), \gamma_{B}(x)\right\}, \theta_{A \cap B}(x)=\min \left\{\theta_{A}(x), \theta_{B}(x)\right\} \forall x \in$ $U$.

\section{- The complement of A:}

$$
\sim A=\left\{\left(x, \gamma_{A}(x), \mu_{A}(x), 1-\theta_{A}(x)\right) \mid \forall x \in U\right\}
$$

Definition 4. Let $U$ and $V$ be two universes and let $A=\left\{\left(x, \mu_{A}(x), \gamma_{A}(x), \theta_{A}(x)\right) \mid x \in U\right\} \quad$ and $B=$ $\left\{\left(y, \mu_{B}(y), \gamma_{B}(y), \theta_{B}(y)\right) \mid y \in V\right\}$ be two SIF sets on $U$ and $V$, respectively. We define the Cartesian product of these two SIF sets

- $A \times B=\left\{\left((x, y), \mu_{A \times B}(x, y)\right.\right.$, $\left.\left.\gamma_{A \times B}(x, y), \theta_{A \times B}(x, y)\right) \mid x \in U, y \in V\right\}$

where $\mu_{A \times B}(x, y)=\mu_{A}(x) \mu_{B}(y), \quad \gamma_{A \times B}(x, y)=$ $\gamma_{A}(x) \gamma_{B}(y), \theta_{A \times B}(x, y)=\theta_{A}(x) \theta_{B}(y) \forall x \in U, y \in V$.

- $A \otimes B=\left\{\left((x, y), \mu_{A \otimes B}(x, y)\right.\right.$,

$\left.\left.\gamma_{A \otimes B}(x, y), \theta_{A \otimes B}(x, y)\right) \mid x \in U, y \in V\right\}$

where $\mu_{A \otimes B}(x, y)=\min \left\{\mu_{A}(x), \mu_{B}(y)\right\}, \gamma_{A \otimes B}(x, y)=$ $\max \left\{\gamma_{A}(x), \gamma_{B}(y)\right\}$

$\theta_{A \otimes B}(x)=\min \left\{\theta_{A}(x), \theta_{B}(y)\right\} \forall x \in U, y \in V$.

- $A \bigotimes B=\left\{\left((x, y), \mu_{A \bigotimes B}(x, y)\right.\right.$,

$\left.\left.\gamma_{A \bigotimes B}(x, y), \theta_{A \bigotimes B}(x, y)\right) \mid x \in U, y \in V\right\}$

where $\mu_{A \bigotimes B}(x, y)=\max \left\{\mu_{A}(x), \mu_{B}(y)\right\}, \gamma_{A \bigotimes B}(x, y)=$ $\min \left\{\gamma_{A}(x), \gamma_{B}(y)\right\}$

$\theta_{A \otimes B}(x)=\max \left\{\theta_{A}(x), \theta_{B}(y)\right\} \forall x \in U, y \in V$.

These definitions are valid, i.e, $A \times B, A \otimes B$, and $A \bigotimes B$ are SIF sets on $U \times V$. Indeed,

For $\quad A \times B \quad: \quad 0 \leq \mu_{A \times B}(x, y)+\gamma_{A \times B}(x, y)=$ $\mu_{A}(x) \mu_{B}(y)+\gamma_{A}(x) \gamma_{B}(y) \leq \mu_{A}(x)+\gamma_{A}(x) \leq 1, \forall x \in$ $U, y \in V$ and $0 \leq \theta_{A \times B}(x, y)=\theta_{A}(x) \theta_{B}(y) \leq 1$, $\forall x \in U, y \in V$.

+ For $A \otimes B$ : If $\forall x \in U, y \in V, \gamma_{A \otimes B}(x, y)=$ $\max \left\{\gamma_{A}(x), \gamma_{B}(y)\right\}=\gamma_{B}(y)$ then $0 \leq \mu_{A \otimes B}(x, y)+$ $\gamma_{A \otimes B}(x, y)=$

$\min \left\{\mu_{A}(x), \mu_{B}(y)\right\}+\max \left\{\gamma_{A}(x), \gamma_{B}(y)\right\} \leq \mu_{B}(y)+$ $\gamma_{B}(y) \leq 1, \forall x \in U, y \in V$.

Obviously, $\quad 0 \leq \theta_{A \otimes B}(x)=\min \left\{\theta_{A}(x), \theta_{B}(y)\right\} \leq$ $1 \forall x \in U, y \in V$.

+ Similarity, $A \otimes B$ is a SIF set on $U \times V$.

Example 1. Given $A=\frac{(0.8,0.1,0.7)}{u_{1}}+\frac{(0.7,0.2,0.6)}{u_{2}}+$ $\frac{(0.5,0.4,0.9)}{u_{3}}$ and $B=\frac{(0.5,0.3,0.2)}{u_{1}}+\frac{(0.6,0.1,0.8)}{u_{2}}+\frac{(0.7,0.2,0.7)}{u_{3}}$ are support-intuitionistic fuzzy set on the universe $U=$ $\left\{u_{1}, u_{2}, u_{3}\right\}$. Then

$$
\begin{aligned}
& A \cap B=\frac{(0.5,0.3,0.2)}{u_{1}}+\frac{(0.6,0.2,0.6)}{u_{2}}+\frac{(0.5,0.4,0.7)}{u_{3}} \\
& A \cup B=\frac{(0.8,0.1,0.7)}{u_{1}}+\frac{(0.7,0.1,0.8)}{u_{2}}+\frac{(0.7,0.2,0.9)}{u_{3}}
\end{aligned}
$$




$$
\begin{aligned}
& \sim A=\frac{(0.1,0.8,0.3)}{u_{1}}+\frac{(0.2,0.7,0.4)}{u_{2}}+\frac{(0.4,0.5,0.9)}{u_{3}} \\
& A \otimes B=\frac{(0.5,0.3,0.2)}{\left(u_{1}, u_{1}\right)}+\frac{(0.6,0.1,0.7)}{\left(u_{1}, u_{2}\right)}+\frac{(0.7,0.2,0.7)}{\left(u_{1}, u_{3}\right)} \\
& +\frac{(0.5,0.3,0.6)}{\left(u_{2}, u_{1}\right)}+\frac{(0.6,0.2,0.6)}{\left(u_{2}, u_{2}\right)}+\frac{(0.7,0.2,0.6)}{\left(u_{2}, u_{3}\right)}+\frac{(0.5,0.4,07)}{\left(u_{3}, u_{1}\right)} \\
& +\frac{(0.5,0.4,0.8)}{\left(u_{3}, u_{2}\right)}+\frac{(0.5,0.4,0.7)}{\left(u_{3}, u_{3}\right)}
\end{aligned}
$$

Now, we consider some properties of the defined operators on $\operatorname{SIFS}(U)$.

Proposition 1. For all $A, B, C \in \operatorname{SIFS}(U)$, then

(a) If $A \subseteq B$ and $B \subseteq C$ then $A \subseteq C$,

(b) $\sim(\sim A)=A$,

(c) Operators $\cap$ and $\cup$ are commutative, associative, and distributive,

(d) Operators $\cap, \sim$ and $\cup$ satisfy the law of De Morgan.

Proof.

(a), (b) is obviously.

(c) the properties of commutative, associative is easy to verify. We consider the property of distributive:

$$
A \cup(B \cap C)=(A \cup B) \cap(A \cup C)
$$

and

$$
A \cap(B \cup C)=(A \cap B) \cup(A \cap C)
$$

Indeed, for each $x \in U$ :

$+\mu_{A \cup(B \cap C)}(x)=\max \left\{\mu_{A}(x), \mu_{B \cap C}(x)\right\}=$ $\max \left\{\mu_{A}(x), \min \left\{\mu_{B}(x), \mu_{C}(x)\right\}\right\}=$ $\min \left\{\max \left\{\mu_{A}(x), \mu_{B}(x)\right\}\right.$,

$\left.\max \left\{\mu_{A}(x), \mu_{C}(x)\right\}\right\}=\min \left\{\mu_{A \cup B}(x), \mu_{A \cup C}(x)\right\}=$ $\mu_{(A \cup B) \cap(A \cup)}(x)$.

$+\gamma_{A \cup(B \cap C)}(x)=\min \left\{\gamma_{A}(x), \gamma_{B \cap C}(x)\right\}=$ $\min \left\{\gamma_{A}(x), \max \left\{\gamma_{B}(x), \gamma_{C}(x)\right\}\right\}=$

$\max \left\{\min \left\{\gamma_{A}(x), \gamma_{B}(x)\right\}\right.$,

$\left.\min \left\{\gamma_{A}(x), \gamma_{C}(x)\right\}\right\}=\max \left\{\gamma_{A \cup B}(x), \gamma_{A \cup C}(x)\right\}=$ $\gamma_{(A \cup B) \cap(A \cup)}(x)$.

$$
+\theta_{A \cup(B \cap C)}(x)=\max \left\{\theta_{A}(x), \theta_{B \cap C}(x)\right\}=
$$

$\max \left\{\theta_{A}(x), \min \left\{\theta_{B}(x), \theta_{C}(x)\right\}\right\}=$

$\min \left\{\max \left\{\theta_{A}(x), \theta_{B}(x)\right\}\right.$,

$\left.\max \left\{\theta_{A}(x), \theta_{C}(x)\right\}\right\}=\min \left\{\theta_{A \cup B}(x), \theta_{A \cup C}(x)\right\}=$ $\theta_{(A \cup B) \cap(A \cup)}(x)$.

Hence $A \cup(B \cap C)=(A \cup B) \cap(A \cup C)(1)$.

Similarity, we obtain

$$
A \cap(B \cup C)=(A \cap B) \cup(A \cap C)
$$

(d) De Morgan's laws $\sim(A \cup B)=(\sim A) \cap(\sim B)$ (3) and $\sim(A \cap B)=(\sim A) \cup(\sim B)(4)$. Indeed, for each $x \in U$ :

$$
\begin{gathered}
\mu_{\sim(A \cup B)}(x)=\gamma_{A \cup B}(x)=\min \left\{\gamma_{A}(x), \gamma_{B}(x)\right\} \\
=\min \left\{\mu_{\sim A}(x), \mu_{\sim B}(x)\right\}=\mu_{(\sim A) \cap(\sim B)}(x) . \\
\gamma_{\sim(A \cup B)}(x)=\mu_{A \cup B}(x)=\max \left\{\mu_{A}(x), \mu_{B}(x)\right\}= \\
\max \left\{\gamma_{\sim A}(x), \gamma_{\sim B}(x)\right\}=\gamma_{(\sim A) \cap(\sim B)}(x) . \\
\theta_{\sim(A \cup B)}(x)=1-\theta_{A \cup B}(x)=1-\max \left\{\theta_{A}(x), \theta_{B}(x)\right\} \\
=\min \left\{1-\theta_{A}(x), 1-\quad \theta_{B}(x)\right\}= \\
\min \left\{\theta_{\sim A}(x), \theta_{\sim B}(x)\right\}=\theta_{(\sim A) \cap(\sim B)}(x) . \\
\text { Hence } \sim(A \cup B)=(\sim A) \cap(\sim B)(3) .
\end{gathered}
$$

Similarity, we obtain $\sim(A \cap B)=(\sim A) \cup(\sim B)(4)$. $\square$ Proposition 2. For every three universes $X, Y, Z$ and four SIF sets $A, B$ on $X, C$ on $Y, D$ on $Z$, we have

(a) $A \times C=C \times A$

(b) $(A \times C) \times D=A \times(C \times D)$

(c) $(A \cup B) \times C=(A \times C) \cup(B \times C)$

(d) $(A \cap B) \times C=(A \times C) \cap(B \times C)$

Proof.

(a), (b) is obviously.

(c) We have $\mu_{(A \cup B) \times C}(x, y)=\mu_{A \cup B}(x) \mu_{C}(y)=$ $\max \left\{\mu_{A}(x), \mu_{B}(x)\right\} \mu_{C}(y)=\max \left\{\mu_{A}(x) \mu_{C}(y)\right.$, $\left.\mu_{B}(x) \mu_{C}(y)\right\}=\max \left\{\mu_{A \cup C}(x, y), \mu_{B \cup C}(x, y)\right\}=$ $\mu_{(A \times C) \cup(B \times C)}(x, y)$,

$\gamma_{(A \cup B) \times C}(x, y)=\gamma_{A \cup B}(x) \mu_{C}(y)=\min \left\{\gamma_{A}(x)\right.$, $\left.\gamma_{B}(x)\right\} \gamma_{C}(y)=\min \left\{\gamma_{A}(x) \gamma_{C}(y), \gamma_{B}(x) \gamma_{C}(y)\right\}=$ $\min \left\{\gamma_{A \cup C}(x, y), \gamma_{B \cup C}(x, y)\right\}=\gamma_{(A \times C) \cup(B \times C)}(x, y)$,

$\theta_{(A \cup B) \times C}(x, y)=\theta_{A \cup B}(x) \theta_{C}(y)=$ $\max \left\{\theta_{A}(x), \theta_{B}(x)\right\} \theta_{C}(y)=\max \left\{\theta_{A}(x) \theta_{C}(y)\right.$, $\left.\theta_{B}(x) \theta_{C}(y)\right\}=\max \left\{\theta_{A \cup C}(x, y), \theta_{B \cup C}(x, y)\right\}=$ $\theta_{(A \times C) \cup(B \times C)}(x, y)$.

It means $(A \cup B) \times C=(A \times C) \cup(B \times C)$. Similarity, $(A \cap B) \times C=(A \times C) \cap(B \times C)$. $\square$

Proposition 3. For every three universes $X, Y, Z$ and four SIF sets $A, B$ on $X, C$ on $Y, D$ on $Z$, we have

(a) $A \otimes C=C \otimes A$

(b) $(A \otimes C) \otimes D=A \otimes(C \otimes D)$

(c) $(A \cup B) \otimes C=(A \otimes C) \cup(B \otimes C)$

(d) $(A \cap B) \otimes C=(A \otimes C) \cap(B \otimes C)$

Proof.

(a), (b) is obviously.

(c) We have $\mu_{(A \cup B) \otimes C}(x, y)=\min \left\{\mu_{A \cup B}(x), \mu_{C}(y)\right\}=$ $\min \left\{\max \left\{\mu_{A}(x), \mu_{B}(x)\right\}, \mu_{C}(y)\right\}=$ $\max \left\{\min \left\{\mu_{A}(x), \mu_{C}(y)\right\}, \min \left\{\mu_{A}(x), \mu_{C}(y)\right\}\right\}=$ $\max \left\{\mu_{A \otimes C}(x, y), \mu_{B \otimes C}(x, y)\right\}=\mu_{A \otimes C \cup B \otimes C}(x, y)$

$\gamma_{(A \cup B) \otimes C}(x, y)=\max \left\{\gamma_{A \cup B}(x), \gamma_{C}(y)\right\}=\max$ $\left\{\min \left\{\gamma_{A}(x), \gamma_{B}(x)\right\}, \gamma_{C}(y)\right\}=\min \left\{\max \left\{\gamma_{A}(x), \gamma_{C}(y)\right\}\right.$, $\left.\max \left\{\gamma_{A}(x), \gamma_{C}(y)\right\}\right\}=\min \left\{\gamma_{A \otimes C}(x, y), \gamma_{B \otimes C}(x, y)\right\}=$ $\gamma_{A \otimes C \cup B \otimes C}(x, y)$

$$
\theta_{(A \cup B) \otimes C}(x, y)=\min \left\{\theta_{A \cup B}(x), \theta_{C}(y)\right\}=\min
$$

$\left\{\max \left\{\theta_{A}(x), \theta_{B}(x)\right\}, \theta_{C}(y)\right\}=\max \left\{\min \left\{\theta_{A}(x), \theta_{C}(y)\right\}\right.$, $\left.\min \left\{\theta_{A}(x), \theta_{C}(y)\right\}\right\}=\max \left\{\theta_{A \otimes C}(x, y), \theta_{B \otimes C}(x, y)\right\}=$

$\theta_{A \otimes C \cup B \otimes C}(x, y)$.

Hence $\quad(A \cup B) \otimes C=(A \otimes C) \cup(B \otimes C)$ Similarity, $(A \cap B) \otimes C=(A \otimes C) \cap(B \otimes C)$. $\square$

In the same as Proposition 3, we get

Proposition 4. For every three universes $X, Y, Z$ and four SIF sets $A, B$ on $X, C$ on $Y, D$ on $Z$, we have

(a) $A \bigotimes C=C \bigotimes A$

(b) $(A \bigotimes C) \bigotimes D=A \bigotimes(C \bigotimes D)$

(c) $(A \otimes B) \bigotimes C=(A \bigotimes C) \cup(B \otimes C)$

(d) $(A \cap B) \bigotimes C=(A \bigotimes C) \cap(B \bigotimes C)$

\section{DistanCE BETWEEN SUPPORT-INTUITIONISTIC FUZZY SETS}

Distance between fuzzy sets and distance between intuitionistic fuzzy set were defined in fuzzy literature 
and have been applied in various problems [4][9]. In this section some extensions of distance between supportintuitionistic fuzzy set in the sense of Szmidt and Kacprzyk [6] are presented.

Definition 5. Let $U=\left\{u_{1}, u_{2}, \ldots, u_{n}\right\}$ be the universe set. $A=\left\{\left(x, \mu_{A}(x), \gamma_{A}(x), \theta_{A}(x)\right) \mid x \in U\right\} \quad$ and $\quad B=$ $\left\{\left(x, \mu_{B}(x), \gamma_{B}(x), \theta_{B}(x)\right) \mid x \in U\right\}$ be two SIF sets on $U$, then distances for $A$ and $B$ are:

- The Hamming distance $d_{S I}(A, B)$

$$
\begin{aligned}
d_{S I}(A, B)=\sum_{i=1}^{n}( & \left|\mu_{A}\left(u_{i}\right)-\mu_{B}\left(u_{i}\right)\right|+\left|\gamma_{A}\left(u_{i}\right)-\gamma_{B}\left(u_{i}\right)\right| \\
& \left.+\left|\theta_{A}\left(u_{i}\right)-\theta_{B}\left(u_{i}\right)\right|\right)
\end{aligned}
$$

- The Euclidean distance $e_{P}(A, B)$

$$
\begin{aligned}
e_{S I}(A, B)=\left(\sum_{i=1}^{n}\right. & \left(\left|\mu_{A}\left(u_{i}\right)-\mu_{B}\left(u_{i}\right)\right|^{2}\right. \\
& +\left|\gamma_{A}\left(u_{i}\right)-\gamma_{B}\left(u_{i}\right)\right|^{2} \\
& \left.\left.+\left|\theta_{A}\left(u_{i}\right)-\theta_{B}\left(u_{i}\right)\right|^{2}\right)\right)^{\frac{1}{2}}
\end{aligned}
$$

Example 2. Given $A=\frac{(0.8,0.1,0.7)}{u_{1}}+\frac{(0.7,0.2,0.6)}{u_{2}}+$ $\frac{(0.5,0.4,0.9)}{u_{3}}$ and $B=\frac{(0.5,0.3,0.2)}{u_{1}}+\frac{(0.6,0.1,0.8)}{u_{2}}+\frac{(0.7,0.2,0.7)}{u_{3}}$ are support-intuitionistic fuzzy set on the universe $U=$ $\left\{u_{1}, u_{2}, u_{3}\right\}$. Thend $d_{S I}(A, B)=2, e_{S I}(A, B)=\sqrt{0.56}$.

\section{SUPPORT-INTUITIONISTIC FUZZY RELATION}

\section{A. Support-intuitionistic fuzzy relation}

Fuzzy relations are one of most important notions of fuzzy set theory and fuzzy systems theory. The Zadeh' composition rule of inference [9] is a well-known method in approximation theory and inference methods in fuzzy control theory. Intuitionistic fuzzy relations were also received many results by researches [4],[5] and [8]. In the same as the construction of fuzzy relations in fuzzy set theory, support-intuitionistic fuzzy relationships building on support-intuitionistic fuzzy set theory, as an extension of fuzzy relation and intuitionistic fuzzy relation, has important implications for both theory and applications. In this section, we shall present some preliminary results on support-intuitionistic fuzzy relations.

Let $X, Y$, and $Z$ be crisp non-empty sets.

Definition 5.[4]. An intuitionistic fuzzy relation $R$ from $U$ to $V$ is an intuitionistic fuzzy subset of $U \times V$, i.e, is an expression given by

$$
R=\left\{\left((x, y), \mu_{R}(x, y), \gamma_{R}(x, y)\right) \mid(x, y) \in U \times V\right\}
$$

where $\mu_{R}, \gamma_{R}: U \times V \rightarrow[0,1]$ such that $\mu_{R}(x, y)+$ $\gamma_{R}(x, y) \leq 1$, for all $(x, y) \in U \times V$.

The most important properties of intuitionistic fuzzy relations were studied in [4],[5].

Now, we define a support-intuitionistic fuzzy relation as an extension of intuitionistic fuzzy relation.

Definition 6. A support-intuitionistic fuzzy relation $R$ from $U$ to $V$ is a support-intuitionistic fuzzy subset of $U \times V$, i.e, is an expression given by

$$
R=\left\{\left((x, y), \mu_{R}(x, y), \gamma_{R}(x, y), \theta_{R}(x, y)\right) \mid(x, y) \in\right.
$$

$U \times V\}$

where $\mu_{R}, \gamma_{R}: U \times V \rightarrow[0,1]$ such that $\mu_{R}(x, y)+$ $\gamma_{R}(x, y) \leq 1$ and $\theta_{R}: U \times V \rightarrow[0,1]$ for all $(x, y) \in U \times$ $V$.

We denote SIFR $(U \times V)$ to be the family of supportintuitionistic fuzzy relation $\mathrm{R}$ from $\mathrm{U}$ to $\mathrm{V}$

As same as representation of fuzzy relation [6], we can represent the support-intuitionistic fuzzy relations by using the graphs, relational tables, matrices.

We denote $\operatorname{SIFR}(U \times V)$ to be the set of all the support-intuitionistic fuzzy relation on $U \times V$.

Definition 7. Given $R \in \operatorname{SIFR}(U \times V)$. We define the inverse relation $R^{-1}$ from $V$ to $U$ as follows:

$$
R^{-1}=\left\{\left((x, y), \mu_{R^{-1}}(y, x), \gamma_{R^{-1}}(y, x), \theta_{R^{-1}}(y, x)\right) \mid\right.
$$
$(y, x) \in V \times U\}$,

where $\mu_{R^{-1}}(y, x)=\mu_{R}(x, y), \gamma_{R^{-1}}(y, x)=\gamma_{R}(x, y)$ and $\theta_{R^{-1}}(y, x)=\theta_{R}(x, y)$ for all $(y, x) \in V \times U$.

This definition is true, because $\mu_{R^{-1}}(y, x)+$ $\gamma_{R^{-1}}(y, x)=\mu_{R}(x, y)+\gamma_{R}(x, y) \leq 1 \forall(y, x) \in V \times$ $U$ and $\mu_{R^{-1}}, \gamma_{R^{-1}}, \theta_{R^{-1}}: V \times U \rightarrow[0,1]$.

Moreover, it is easy to verify the true of the following concepts:

Definition 8. Let $R$ and $P$ be two support-intuitionistic fuzzy relations between $U$ and $V$, for every $(x, y) \in U \times$ $V$ we can define:

- $R \leq P$ iff $\left\{\begin{array}{l}\mu_{R}(x, y) \leq \mu_{P}(x, y) \\ \gamma_{R}(x, y) \geq \gamma_{P}(x, y) \\ \theta_{R}(x, y) \leq \theta_{P}(x, y)\end{array}\right.$

- $R \vee P=\left\{\left((x, y), \mu_{R \vee P}(x, y), \gamma_{R \vee P}(x, y)\right.\right.$,

$\left.\left.\theta_{R \vee P}(x, y)\right) \mid(x, y) \in U \times V\right\}$, where

$$
\begin{gathered}
\mu_{R \vee P}(x, y)=\max \left\{\mu_{R}(x, y), \mu_{P}(x, y)\right\}, \\
\gamma_{R \vee P}(x, y)=\min \left\{\gamma_{R}(x, y), \gamma_{R}(x, y)\right\}, \\
\theta_{R \vee P}(x, y)=\max \left\{\theta_{R}(x, y), \theta_{R}(x, y)\right\},
\end{gathered}
$$

- $R \wedge P=\left\{\left((x, y), \mu_{R \wedge P}(x, y), \gamma_{R \wedge P}(x, y)\right.\right.$,

$\left.\left.\theta_{R \wedge P}(x, y)\right) \mid(x, y) \in U \times V\right\}$, where

$$
\begin{aligned}
& \mu_{R \wedge P}(x, y)=\min \left\{\mu_{R}(x, y), \mu_{P}(x, y)\right\}, \\
& \gamma_{R \wedge P}(x, y)=\max \left\{\gamma_{R}(x, y), \gamma_{P}(x, y)\right\}, \\
& \theta_{R \wedge P}(x, y)=\min \left\{\theta_{R}(x, y), \theta_{P}(x, y)\right\},
\end{aligned}
$$

- $\sim R=\left\{\left((x, y), \mu_{\sim R}(x, y), \gamma_{\sim R}(x, y)\right.\right.$,

$\left.\left.\theta_{\sim R}(x, y)\right) \mid(x, y) \in U \times V\right\}$, where

$$
\mu_{\sim R}(x, y)=\gamma_{R}(x, y), \gamma_{\sim R}(x, y)=\mu_{R}(x, y)
$$

and $\theta_{\sim R}(x, y)=1-\theta_{R}(x, y)$

Proposition 5. Let $R, P, Q \in \operatorname{SIFR}(U \times V)$. Then

(a) $\left(R^{-1}\right)^{-1}=R$

(b) $R \leq P \Rightarrow R^{-1} \leq P^{-1}$

(c) $(R \wedge P)^{-1}=R^{-1} \wedge P^{-1}$ and

$$
(R \vee P)^{-1}=R^{-1} \vee P^{-1}
$$

(d) $R \wedge(P \vee Q)=(R \wedge P) \vee(R \wedge Q)$ and 


$$
R \vee(P \wedge Q)=(R \vee P) \wedge(R \vee Q)
$$

(e) $R \wedge P \leq R, \quad R \wedge P \leq P$

(f) If $R \geq P$ and $R \geq Q$ then $R \geq P \vee Q$

(g) If $R \leq P$ and $R \leq Q$ then $R \leq P \wedge Q$.

\section{Proof.}

(a),(b), (c), (e), (f), (g) are obviously.

(d) Expression $R \wedge(P \vee Q)=(R \wedge P) \vee(R \wedge Q)$ is true, because we have

$+\mu_{R \wedge(P \vee Q)}(x, y)=\min \left\{\mu_{R}(x, y), \mu_{P \vee Q}(x, y)\right\}=$ $=\min \left\{\mu_{R}(x, y), \max \left\{\mu_{P}(x, y), \mu_{Q}(x, y)\right\}\right\}=\max$ $\left\{\min \left\{\mu_{R}(x, y), \mu_{P}(x, y)\right\}, \min \left\{\mu_{R}(x, y), \mu_{Q}(x, y)\right\}=\right.$ $\max \left\{\mu_{R \wedge P}(x, y), \mu_{R \wedge Q}(x, y)\right\}$

$=\mu_{(R \wedge P) \vee(R \wedge Q)}(x, y)$

$+\gamma_{R \wedge(P \vee Q)}(x, y)=\max \left\{\gamma_{R}(x, y), \gamma_{(P \vee Q)}(x, y)\right\}=$ $=\max \left\{\gamma_{R}(x, y), \min \left\{\gamma_{P}(x, y), \gamma_{Q}(x, y)\right\}=\min \right.$ $\left\{\max \left\{\gamma_{R}(x, y), \gamma_{P}(x, y)\right\}, \max \left\{\gamma_{R}(x, y), \gamma_{Q}(x, y)\right\}\right\}=$ $=\min \left\{\gamma_{R \wedge P}(x, y), \gamma_{R \wedge Q}(x, y)\right\}$

$=\gamma_{(R \wedge P) \vee(R \wedge Q)}(x, y)$

$+\theta_{R \wedge(P \vee Q)}(x, y)=\min \left\{\theta_{R}(x, y), \theta_{P \vee Q}(x, y)\right\}=$ $\min \left\{\theta_{R}(x, y), \max \left\{\theta_{P}(x, y), \theta_{Q}(x, y)\right\}\right\}=\max$ $\left\{\min \left\{\theta_{R}(x, y), \theta_{P}(x, y)\right\}, \min \left\{\theta_{R}(x, y), \theta_{Q}(x, y)\right\}=\right.$ $\max \left\{\theta_{R \wedge P}(x, y), \theta_{R \wedge Q}(x, y)\right\}$

$=\theta_{(R \wedge P) \vee(R \wedge Q)}(x, y)$

Similarity, the expression $R \vee(P \wedge Q)=(R \vee P) \wedge$ $(R \vee Q)$ is true.

\section{B. Composition of support-intuitionistic fuzzy relations}

Definition 9. Let $P \in \operatorname{SIFR}(U \times V)$ and $Q \in \operatorname{SIFR}(V \times$ $W)$. Then, the max-min composition of the supportintuitionistic fuzzy relation $P$ with the supportintuitionistic fuzzy relation $Q$ is a fuzzy relation $P \circ Q$ on $U \times W$ which as define by:

$$
\begin{aligned}
& \mu_{P \circ Q}(x, z)=\max _{y \in V}\left\{\min \left\{\mu_{P}(x, y), \mu_{Q}(y, z)\right\}\right\} \\
& \gamma_{P \circ Q}(x, z)=\min _{y \in V}\left\{\max \left\{\gamma_{P}(x, y), \gamma_{Q}(y, z)\right\}\right\} \\
& \theta_{P \circ Q}(x, z)=\max _{y \in V}\left\{\min \left\{\theta_{P}(x, y), \theta_{Q}(y, z)\right\}\right\}
\end{aligned}
$$

Example 3. Let $P \in \operatorname{SIFR}(U \times V)$ and $Q \in \operatorname{SIFR}(V \times$ $W)$ be two support-intuitionistic fuzzy relation from $U=\{x 1, x 2\}$ to $V=\{y 1, y 2, y 3\}$ and from $V$ to $W=$ $\{z 1, z 2\}$, respectively

\begin{tabular}{|c|c|c|c|}
\hline $\mathrm{P}$ & $\mathrm{y} 1$ & $\mathrm{y} 2$ & $\mathrm{y} 3$ \\
\hline $\mathrm{x} 1$ & $0.5,0,2,0.7$ & $0.7,0.2,0.5$ & $0.8,0.1,0.9$ \\
\hline $\mathrm{x} 2$ & $0.6,0.4,0.8$ & $0.7,0.1,0.4$ & $0.7,0.2,0.8$ \\
\hline
\end{tabular}

and

\begin{tabular}{|c|c|c|}
\hline $\mathrm{Q}$ & $\mathrm{z} 1$ & $\mathrm{z} 2$ \\
\hline $\mathrm{y} 1$ & $0.8,0.1,0.7$ & $0.6,0.2,0.7$ \\
\hline $\mathrm{y} 2$ & $0.1,0.6,0.9$ & $0.8,0.1,0.8$ \\
\hline $\mathrm{y} 3$ & $0.4,0.5,0.8$ & $0.7,0.2,0.9$ \\
\hline
\end{tabular}

The support-intuitionistic fuzzy relations $P \circ Q$ is

\begin{tabular}{|c|c|c|}
\hline$P \circ Q$ & $\mathrm{z} 1$ & $\mathrm{z} 2$ \\
\hline $\mathrm{x} 1$ & $0.5,0.2,0.8$ & $0.7,0.2,0.9$ \\
\hline $\mathrm{x} 2$ & $0.5,0.4,0.8$ & $0.7,0.1,0.8$ \\
\hline
\end{tabular}

Proposition 6. The fuzzy relation $P \circ Q$ on $U \times W$ in Definition 9 is the support-intuitionistic fuzzy relation from $U$ to $W$.

Proof.

Indeed, it always exists an element $y_{1} \in Y$ such that

$$
\begin{aligned}
& \mu_{P \circ Q}(x, z)=\max _{y \in V}\left\{\min \left\{\mu_{P}(x, y), \mu_{Q}(y, z)\right\}\right\} \\
= & \min \left\{\mu_{P}\left(x, y_{1}\right), \mu_{Q}\left(y_{1}, z\right)\right\}
\end{aligned}
$$

and $\max \left\{\gamma_{P}\left(x, y_{1}\right), \gamma_{Q}\left(y_{1}, z\right)\right\}=\gamma_{Q}\left(y_{1}, z\right)$. Then

$0 \leq \mu_{P \circ Q}(x, z)+\gamma_{P \circ Q}(x, z) \leq$

$\min \left\{\mu_{P}\left(x, y_{1}\right), \mu_{Q}\left(y_{1}, z\right)\right\}+\max \left\{\gamma_{P}\left(x, y_{1}\right), \gamma_{Q}\left(y_{1}, z\right)\right\}$

$\leq \mu_{Q}\left(y_{1}, z\right)+\gamma_{Q}\left(y_{1}, z\right) \leq 1$, because $Q \in \operatorname{SIFR}(V \times$ $W)$. Moreover, $P \in \operatorname{SIFR}(U \times V)$ and $Q \in \operatorname{SIFR}(V \times$ $W)$ then $0 \leq \theta_{P \circ Q}(x, z)=\max _{y \in V}\left\{\min \left\{\theta_{P}(x, y), \theta_{Q}(y, z)\right\}\right\} \leq 1$. $\square$

Proposition 7. Let $U, V, W$ be the universe sets. $P, Q$ are SIFRs on $U \times V, V \times W$, respectively. Then $(P \circ Q)^{-1}=$ $Q^{-1} \circ P^{-1}$

Proof. We have

$$
\begin{aligned}
& \quad+\mu_{(P \circ Q)^{-1}}(z, x)=\mu_{P \circ Q}(x, z) \\
& =\max _{y}\left\{\min \left[\mu_{P}(x, y), \mu_{Q}(y, z)\right]\right\} \\
& \quad=\max _{y}\left\{\min \left[\mu_{Q^{-1}}(z, y), \mu_{P^{-1}}(y, x)\right]\right\} \\
& =\mu_{Q^{-1} \circ P^{-1}}(z, x)
\end{aligned}
$$

+ Similarity, $\gamma_{(P \circ Q)^{-1}}(z, x)=\gamma_{Q^{-1} \circ P^{-1}}(z, x)$ and $\theta_{(P \circ Q)^{-1}}(z, x)=\theta_{Q^{-1} \circ P^{-1}}(z, x) \square$

A generalized concept of composition of two supportintuitionistic fuzzy relations will be represent as follows:

Definition 10. Let $P \in \operatorname{SIFR}(U \times V)$ and $Q \in \operatorname{SIFR}(V \times$ $W)$.Let $\alpha=\vee, \beta=\Lambda, T t-$ norm, $S t-$ conorm be $t$ norm an t-conorm, dual two-two on $[0,1]$. Then, the generalized composition of the support-intuitionistic fuzzy relation $P$ with the support-intuitionistic fuzzy relation $Q$ is a fuzzy relation $P{ }^{\circ}{ }_{S, T} Q$ on $U \times W$ which as define by:

$$
\begin{aligned}
& \mu_{P{ }^{\circ}, T}(x, z)=\vee_{y \in V}\left\{T\left\{\mu_{P}(x, y), \mu_{Q}(y, z)\right\}\right\} \\
& \gamma_{P{ }^{\circ} S, T}(x, z)=\wedge_{y \in V}\left\{S\left\{\gamma_{P}(x, y), \gamma_{Q}(y, z)\right\}\right\} \\
& \theta_{P{ }^{\circ}, T Q}(x, z)=\vee_{y \in V}\left\{T\left\{\theta_{P}(x, y), \theta_{Q}(y, z)\right\}\right\}
\end{aligned}
$$

Here, we note that $0 \leq T\left\{\mu_{P}(x, y), \mu_{Q}(y, z)\right\}+$ $S\left\{\mu_{P}(x, y), \mu_{Q}(y, z)\right\} \leq 1, \forall x \in U, y \in V, z \in T$. So, this concepts is well.

\section{Reflexive, symmetric, transitive SIFRs}

In this section, we consider the properties of supportintuitionistic fuzzy relations on a set, such as reflexive, symmetric, transitive properties. 
When $U \equiv V$ then $R \in \operatorname{SIFR}(U \times U)$ is called a support-intuitionistic fuzzy relations on a set $U$ and denoted $R \in \operatorname{SIFR}(U)$.

Definition 11. (i) The support-intuitionistic fuzzy relations $R \in \operatorname{SIFR}(U)$ is called reflexive iff $\mu_{R}(x, x)=$ $\theta_{R}(x, x)=1$ and $\gamma_{R}(x, x)=0$, for all $x \in U$.

(ii) The support-intuitionistic fuzzy relations $R \in$ $\operatorname{SIFR}(U)$ is called anti-reflexive iff $\mu_{R}(x, x)=$ $\theta_{R}(x, x)=0$ and $\gamma_{R}(x, x)=1$, for all $x \in U$.

It is easy to verify two following results.

Proposition 8. If the support-intuitionistic fuzzy relations $R, P \in \operatorname{SIFR}(U)$ is reflexive, then

(a) $R \wedge P, R \vee P$ is also.

(b) $\sim R$ is anti-reflexive.

Proposition 9. If the support-intuitionistic fuzzy relations $R, P \in \operatorname{SIFR}(U)$ is anti-reflexive, then

(a) $R \wedge P, R \vee P$ is also.

(b) $\sim R$ is reflexive.

Definition 12. The support-intuitionistic fuzzy relations $R \in \operatorname{SIFR}(U)$ is called symmetric iff $\mu_{R}(x, y)=\mu_{R}(y, x)$, $\gamma_{R}(x, y)=\gamma_{R}(y, x)$ and $\theta_{R}(x, y)=\theta_{R}(y, x)$ for all $x, y \in U$.

Proposition 10. If the support-intuitionistic fuzzy relations $R, P \in \operatorname{SIFR}(U)$ is symmetric, then $R \wedge P$, $R \vee P, \sim R$ is also.

Definition 13. The support-intuitionistic fuzzy relations $R \in \operatorname{SIFR}(U)$ is called max-min transitive iff $R \circ R \leq R$.

\section{Similarity support-intuitionistic fuzzy relations}

Definition 14. The support-intuitionistic fuzzy relations $R \in \operatorname{SIFR}(U)$ is called similarity if it is reflexive, symmetric and (max-min) transitive.

Example 4. Given $U=\{u 1, u 2\}$. Let $R \in \operatorname{SIFR}(U \times U)$ be a support-intuitionistic fuzzy relation defined as follows.

\begin{tabular}{|c|c|c|}
\hline $\mathrm{R}$ & $\mathrm{u} 1$ & $\mathrm{u} 2$ \\
\hline $\mathrm{u} 1$ & $(1,0,1)$ & $(0,1,0)$ \\
\hline $\mathrm{u} 2$ & $(0,1,0)$ & $(1,0,1)$ \\
\hline
\end{tabular}

It is easy to verify $R$ satisfy properties: reflexive, symmetric, (max-min) transitive. Then $R$ is a similarity support-intuitionistic fuzzy relation.

\section{CONCLUSION}

In this paper, the new notion of support-intuitionistic fuzzy set was introduced, in section 2. Some properties of SIF sets were appeared in section 3 . In section 4 , we give some distances between two SIF sets. Finally, we construct the notions of support-intuitionistic fuzzy relation and investigate initially properties as reflexive, symmetric, transitive of its. In the future, we shall deal with support-intuitionistic fuzzy logic operators and give some applications in decision making problems.

\section{REFERENCES}

[1] L.A. Zadeh, Fuzzy sets, Information and control, vol.8,1965, pp 338-353.

[2] K. Atanassov, Intuitionistic fuzzy sets, Fuzzy set and systems, vol.20, 1986, pp.87-96.

[3] K. Atanassov, More on intuitionistic fuzzy sets, Fuzzy Sets and Systems, vol.33 ,1989, pp. 37-45.

[4] P. Burilo and H. Bustince, Intuitionistic fuzzy relations (Part I), Mathware and soft computing, vol. 2, 1995, pp 538.

[5] H. Bustince, Intuitionistic fuzzy relation with predetermined properties, Fuzzy sets and systems, vol. 109, 1996, pp 379-403.

[6] E. Smidt and J. Kacpryk, Distance between intuitionistic fuzzy set, Fuzzy sets and systems, vol. 114, 2000, pp 505518.

[7] W. B. V. Kandasamy and F. Smarandache, Fuzzy relational maps and neutrosophic relational maps, HEXIS Church Rock, 2004.

[8] H.L Yang, S. G. Li, Restudy of Intuitionistic Fuzzy Relations, Systems Engineering - Theory \& Practice, Volume 29, Issue 2, February 2009, pp 114-120.

[9] J. Fodor and M. Roubens, Fuzzy preference modeling and multi-criteria decision support, Kluwer Academic Pub., London, 1994.

[10] S. K. De, R. Biswas and A. R. Roy, Some operators on intuitionistic fuzzy sets, Fuzzy set and Systems, Vol. 114, issue 3, 2000, pp $477-484$.

[11] Z. Xu, M. Xia, Induced generalized intuitionistic fuzzy operators, Knowledge-Based Systems, Vol. 24, issue 2, 2011, pp 197-209.

[12] Y. Xiao and et al, A new kind of the intuitionistic fuzzy implication, Journal of Information. \& Computational Sciences, Vol. 8 (13), 2011, pp 2839-2849.

\section{Authors' Profile}

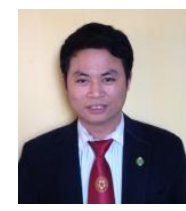

X.T. Nguyen was born on October 28, 1982, in Thai Binh, Viet Nam. He received the B.Sc. and M.S Degrees, in Mathematic from The College of Science, Vietnamese National University (VNU), Hanoi, in 2004 and 2009, respectively.

Now, he is a lecturer in Department of applied Math-Informatics, Faculty of Information Technology, Vietnam National University of Agriculture (VNUA). He is teaching Calculus, Optimization, Fuzzy logic and application. His research interests are Spectral theory of operator Fuzzy set theory, Rough set theory and application in data mining. His representative published article: Remarks on the spectrum of a compact convex set of compact operators, Journal of Applied Analysis 16 (2010), pp 259-264, and Rough Fuzzy Relation on Two Universal Sets, I.J. Intelligent Systems and Applications, 2014, 04, 49-55.

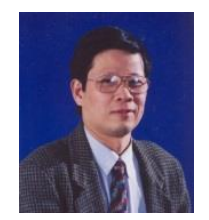

V.D. Nguyen was born on the year 1953 in Hanoi, Vietnam. He was a post-graduate intern in computer Science in Moscow, The Russian Federation. In 2004 he received the Doctor of Philosophy $(\mathrm{PhD})$ Degree in Mathematics at the Vietnam National University (VNU), Hanoi. Now he is an Associate Professor in the Department of applied Maths-Informatics, Faculty of Information Technology, Vietnam National University of Agriculture (VNUA). His representative published article: Rough Fuzzy Relation on Two Universal Sets, I.J. Intelligent Systems and Applications, 2014, 04, 49-55. 